\title{
The driving force and progressive mechanisms of evolution deduced from thermodynamics
}

\author{
Ji-Ming Chen",\# \\ College of Veterinary Medicine, Qingdao Agricultural University \\ ${ }^{*}$ Corresponding author. Email: jmchen678@qq.com; Tel: +86-13854212160. \\ \# ORCID webpage: https://orcid.org/0000-0002-0404-0830
}

\begin{abstract}
Studies on evolution have made significant progress in multiple disciplines, but evolutionary theories remain scattered, complicated, elusive, and controversial. To address this issue, a novel evolutionary theory is deduced from thermodynamics in this article. As per the formula of Gibbs free energy, carbon-based entities (CBEs) on Earth tend to absorb more energy. This is the evolutionary driving force leading to organic synthesis of higherhierarchy CBEs (HHCBEs). The organic synthesis raises the amount of HHCBEs and increases the structural complexity and hierarchy of CBEs. Increased structural complexity and hierarchy spontaneously offer complicated functions to HHCBEs. Genetic mutations, epigenetic changes, and uninheritable variations provide diversified HHCBEs for natural selection which is redefined as survival of the fit and elimination of the unfit, leading to increase of diversity and fitness of HHCBEs. Order in biology resulting from permanent natural selection is largely contrary to order in physics. Natural selection acts on the overall fitness involving all traits through the co-action of positive selection and negative selection. Natural selection can establish biological traits in short geological periods. Different combinations of traits can lead to sympatric speciation targeting the same niche. Altruism, collaboration, and obeying rules with balanced freedom are all important throughout the CBE evolution, which harbors three overlapping phases including chemical evolution (abiogenesis), biological evolution, and group evolution. Altogether, this theory termed the CBE evolutionary theory (CBEET) suggests that evolution which favors fitness and diversity is driven hierarchy-wise by energy. It reveals the driving force of evolution and reestablishes the key role of natural selection. It integrates with advances from multiple disciplines and provides simple and rational answers to some evolutionary conundrums. It removes several elusive or erroneous views including the one regarding negative entropy. It bridges natural sciences and social sciences and sheds novel insights into harmonious development of human society.
\end{abstract}

Keywords: driving force; energy; evolution; fitness; genetic drift; mechanism; natural selection; speciation; thermodynamics; theory

\section{Introduction}

In recent decades, our recognition regarding life origin and biological evolution has been updated greatly with the development of modern biology, geology, chemistry, and astronomy [1-7]. However, these scientific advances have not been well integrated, and multiple concepts, views, and hypotheses pertaining to evolution remain scattered, complicated, elusive, controversial, or erroneous [1-13]. Therefore, it is highly desirable to develop a novel evolutionary theory through synthesis of these advances to interpret most aspects of evolution in a comprehensive and comprehensible way [1-13].

This article aims to deduce such a theory from thermodynamics. It pertains to the evolution of carbon-based entities (CBEs), and is thus termed the CBE evolutionary theory (CBEET). CBEs include small organic molecules (e.g. methane, ethanol), middle organic molecules (e.g. amino acids, nucleotides), large organic molecules (e.g. proteins, nucleic acids, lipids), bacteria, animals, plants, and other organisms. CBEs have hierarchies, and large organic molecules are higher-hierarchy CBEs (HHCBEs) compared with middle organic molecules, but they are lower-hierarchy CBEs (LHCBEs) compared with unicellular organisms. 
The whole evolutionary history of CBEs on Earth harbors three overlapping phases: chemical evolution which is also termed abiogenesis or origin of life, biological evolution, and group evolution which includes development of the societies of ants, bees, and humans.

I published the preliminary version of the CBEET in 2000 as an article and a book from the second law of thermodynamics [1,2]. This preliminary version was deduced from the assumption that the whole universe is an isolated system, without direct connection with the thermodynamic features of the Earth's surface. It was thus relatively abstract and obscure. It did not employ or integrate with research advances in recent 20 years. Through exploration for the past 20 years, the CBEET has been mature from a hypothesis based on an abstract assumption to a theory based on concrete evidence and deduction, and constitutes this article.

The logic model of the CBEET is given in Figure 1. The novelties and advantages of the CBEET compared to previous evolutionary theories or views are given in Table $\mathbf{1}$.

\section{Methods}

\subsection{Logic modelling}

Previous evolutionary theories and views were largely established on the extrapolation logic model shown by the arrows with blue points in Figure 1. This model assumes that regularities applicable to small ranges are also applicable to large ranges, like the methodology that regularities of the evolution within species (microevolution) are assumed to be applicable to the evolution above the species level (macroevolution) [3-5,14]. This model was established on observations regarding the differences, similarities, and relationships in biological traits, structures, functions, fossils, genetics, and epigenetics. They do not rely heavily on energy transformation.

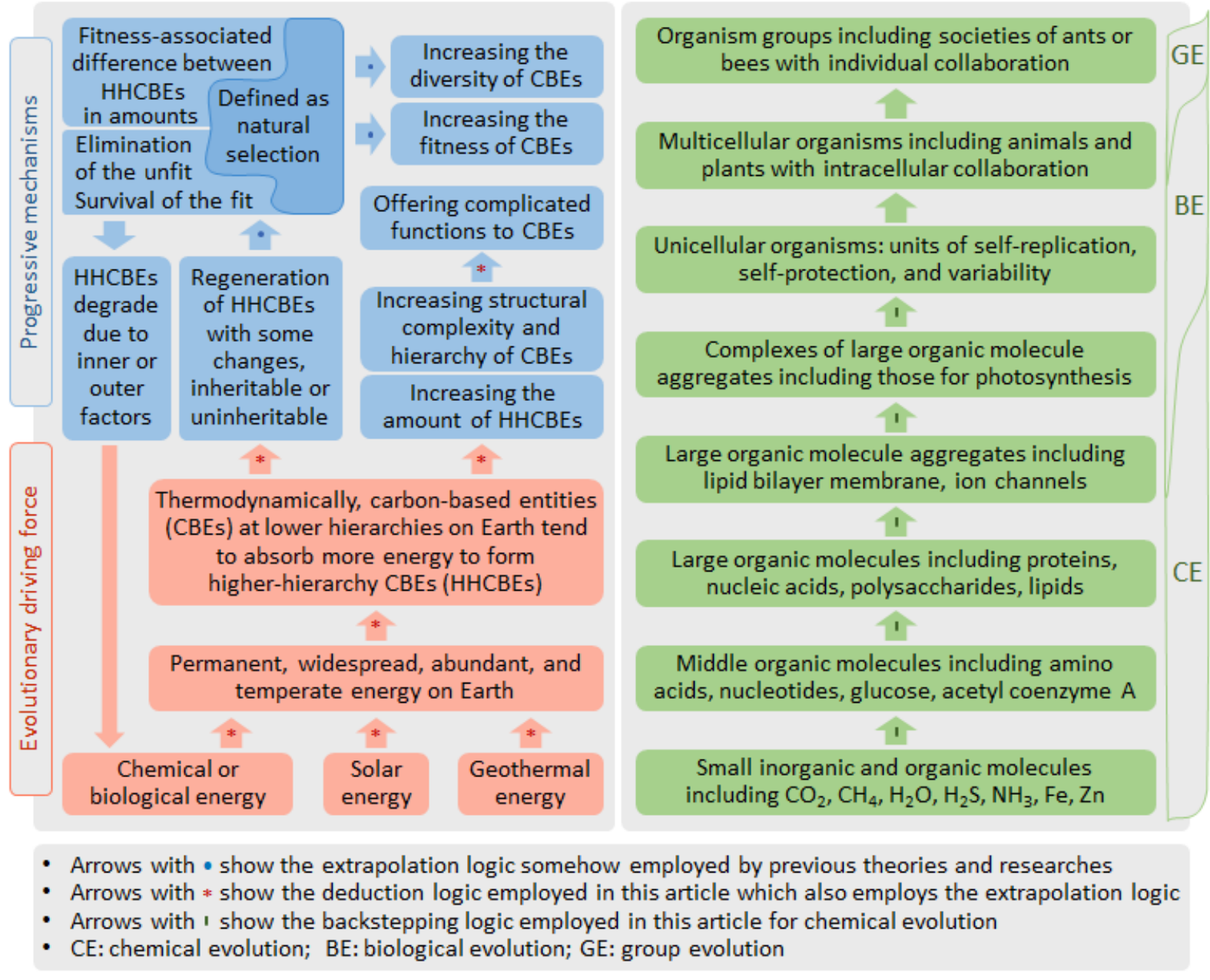

Figure 1. The logic model of the novel evolutionary theory CBEET from the bottom up 
Table 1. Differences and advantages of the novel evolutionary theory CBEET compared to previous theories or views.

\begin{tabular}{|c|c|c|c|}
\hline Topics & Previous theories or views & CBEET views & CBEET advantages \\
\hline Logic model & $\begin{array}{l}\text { Extrapolation (from microevolution to } \\
\text { macroevolution), like exploring a big city } \\
\text { only using a bike to observe each street }\end{array}$ & $\begin{array}{l}\text { Extrapolation and deduction from thermodynamic laws which } \\
\text { are applicable to the ranges broader than evolution, like } \\
\text { exploring a big city using a bike to observe each street and a } \\
\text { helicopter to observe the panorama of the city }\end{array}$ & $\begin{array}{l}\text { (1) More scientific; } \\
\text { (2) More comprehensive and inclusive; } \\
\text { (3) More comprehensible }\end{array}$ \\
\hline $\begin{array}{l}\text { Energy } \\
\text { relevance }\end{array}$ & $\begin{array}{l}\text { With no direct relationship to energy, like } \\
\text { interpreting the running function of a car } \\
\text { without energy consumption }\end{array}$ & $\begin{array}{l}\text { Heavily relied on energy transformation, like interpreting the } \\
\text { running function of a car mainly based on energy consumption }\end{array}$ & $\begin{array}{l}\text { (1) More scientific; } \\
\text { (2) More powerful in explaining } \\
\text { evolutionary myths }\end{array}$ \\
\hline $\begin{array}{l}\text { The driving } \\
\text { force of } \\
\text { evolution }\end{array}$ & $\begin{array}{l}\text { Mistaking natural selection, genetic drift, } \\
\text { mutation, or competition as the driving } \\
\text { force of evolution }\end{array}$ & $\begin{array}{l}\text { As per thermodynamics, the tendency of carbon-based entities } \\
\text { (CBEs) on Earth to absorb more energy is the driving force of } \\
\text { evolution leading to organic synthesis of higher-hierarchy } \\
\text { CBEs (HHCBEs) }\end{array}$ & $\begin{array}{l}\text { Revealing the real driving force of } \\
\text { evolution, which is like a key to a maze }\end{array}$ \\
\hline $\begin{array}{l}\text { The } \\
\text { progressive } \\
\text { mechanisms } \\
\text { of evolution }\end{array}$ & $\begin{array}{l}\text { Natural selection which was defined as } \\
\text { "survival of the fittest" (Charles Darwin's } \\
\text { theory) or "gradual replacement by the } \\
\text { fittest" (the Modern Synthesis) }\end{array}$ & $\begin{array}{l}\text { Three progressive mechanisms: (1) the thermodynamics-driven } \\
\text { organic synthesis increases the amounts of HHCBEs, and } \\
\text { increases the structural complexity and hierarchy of CBEs; (2) } \\
\text { increased structural complexity spontaneously offer } \\
\text { complicated functions to CBEs; (3) natural selection of } \\
\text { HHCBEs carrying various changes leads to increase of } \\
\text { diversity and fitness }\end{array}$ & $\begin{array}{l}\text { (1) More scientific; } \\
\text { (2) More powerful in explaining } \\
\text { evolutionary myths; } \\
\text { (3) Establishing the driving force as the first } \\
\text { leading role in evolution }\end{array}$ \\
\hline $\begin{array}{l}\text { Order in } \\
\text { biology }\end{array}$ & $\begin{array}{l}\text { Assuming that all kinds of order are the } \\
\text { same in nature and equal to low entropy }\end{array}$ & $\begin{array}{l}\text { (1) Order should not be simply hooked with low entropy; } \\
\text { (2) Order in biology showing a moving and warm feature with } \\
\text { increased microstates, is contrary to order in physics which } \\
\text { represents a static and cold feature with reduced microstates }\end{array}$ & $\begin{array}{l}\text { (1) More scientific; } \\
\text { (2) More powerful to bridge physics, } \\
\text { chemistry, and biology }\end{array}$ \\
\hline $\begin{array}{l}\text { Natural } \\
\text { selection }\end{array}$ & $\begin{array}{l}\text { (1) Defined as "survival of the fittest" or } \\
\text { "gradual replacement by the fittest"; } \\
\text { (2) Usually focusing on one trait and } \\
\text { positive selection in one aspect; } \\
\text { (3) Proposing that only inheritable and } \\
\text { random changes under natural selection }\end{array}$ & $\begin{array}{l}\text { (1) Defined as "survival of fit HHCBEs and elimination of } \\
\text { unfit HHCBEs"; } \\
\text { (2) Highlighting the overall fitness constituted by all traits of } \\
\text { HHCBEs and selection from various aspects; } \\
\text { (3) Clarifying that inheritable and uninheritable changes are all } \\
\text { under natural selection; } \\
\text { (4) Clarifying that some changes can be not random }\end{array}$ & $\begin{array}{l}\text { (1) Favoring fitness and diversity; } \\
\text { (2) More inclusive and able to integrate with } \\
\text { research advances in various fields; } \\
\text { (3) Applicable to chemical evolution; } \\
\text { (4) Reflecting the reality better in multiple } \\
\text { aspects }\end{array}$ \\
\hline
\end{tabular}


Chemical evolution

Neither the driving force nor the mechanisms from thermodynamics having been proposed to interpret how large organic molecules evolved into lives

$\begin{array}{ll}\text { Biological } & \text { (1) Interpreting the punctuated } \\ \text { evolution } & \text { equilibrium tempo using geological } \\ \text { isolation; }\end{array}$

(2) Interpreting sympatric speciation with diverging selection targeting different niches in the same area

Group

evolution

(1) Not having established the concept of group evolution and relevant views;

(2) Difficult to explain altruism and social norms directly on an evolutionary basis, and stressing selfishness, competition, and survival of the fittest;

(3) Exerting negative influence on development of human society
(1) Chemical evolution was driven by the force that CBEs on Earth tend to absorb more energy to form HHCBEs;

(2) Lives originated stepwise from small molecules to middle organic molecules, large organic molecule aggregates, and complexes of large organic molecule aggregates

(1) Interpreting the punctuated equilibrium tempo with genetics and natural selection via positive selection and negative selection in multiple aspects, covering the effects of geological isolation and temporal changes;

(2) Proposing a novel sympatric speciation mechanism which targets the same niche in the same area through different combinations of fitness (traits)

(1) Establishing group evolution as a novel phase of the evolution of CBEs;

(2) Clarifying that altruism, collaboration, and obeying rules with balanced freedom are important throughout the evolution of CBEs including group evolution;

(3) Clarifying that struggles are crucial to maintaining animal groups, and altruism and collaboration is critical for winning and avoiding struggles
(1) More scientific;

(2) Describing a rational and

comprehensible mechanism about origin of life

(1) More comprehensive;

(2) More comprehensible;

(3) Reflecting the reality better
(1) Interpreting altruism and collaboration in a scientific and simple way;

(2) Vindicating human rights using evolutionary theories;

(3) Providing constructive suggestions for development of human society;

(4) Bridging the natural evolution and the cultural evolution;

(5) Bridging natural sciences and social sciences

\begin{tabular}{llll}
$\begin{array}{l}\text { General } \\
\text { features }\end{array}$ & $\begin{array}{l}\text { Listlessly, scattered, complicated, elusive, } \\
\text { controversial, entangled with a few } \\
\text { erroneous notions, established on } \\
\text { extrapolation from observations }\end{array}$ & $\begin{array}{l}\text { Energetic, integrated, inclusive, comprehensive, } \\
\text { comprehensible, established on extrapolation from } \\
\text { observations and deduction from thermodynamic laws }\end{array}$ & $\begin{array}{l}\text { (1) More scientific; } \\
\text { (3) More convincing; } \\
\text { comprehensible }\end{array}$ \\
\hline
\end{tabular}


The CBEET was established not only on the extrapolation logic model stated above, but also on the panoramalike deduction logic model shown by the arrows with red asterisks in Figure 1. It employed laws of thermodynamics and other facts listed at Section $\mathbf{2 . 2}$ that are applicable to the ranges larger than evolution, to deduce regularities of evolution. The CBEET is heavily dependent on energy transformation. Regarding chemical evolution, the backstepping logic was employed, as shown by the arrows with green lines in Figure 1. Detailed deduction processes were given at the relevant sites in Sections 3-8.

\subsection{Laws and facts for deduction of the CBEET}

\subsubsection{Laws of thermodynamics $[15,16]$}

There are four laws of thermodynamics. All of them are applicable to all reactions, statuses, and movements of all CBEs on Earth and many other natural processes on Earth.

The zeroth law of thermodynamics is the basis of the other three laws of thermodynamics. It states that if two systems are both in thermal equilibrium with a third system (i.e. they are of the same temperature), then they are in thermal equilibrium with each other.

The first law of thermodynamics is the thermodynamic version of the law of conservation of energy, which states that increase of internal energy of a closed system is equal to the sum of the work the outside gives to the system and the heat the outside gives to the system.

The second law of thermodynamics can be expressed in several ways. One is that heat does not spontaneously pass from a colder body to a warmer body. Another is that the entropy of an isolated system never decreases over time.

The third law of thermodynamics states that the entropy of a system approaches a constant value as its temperature approaches absolute zero, and the entropies of perfect crystalline substances at absolute zero temperature are zero.

\subsubsection{Two formulas of entropy $[15,16]$}

Macroscopically and thermodynamically, changes of entropy (dS) of a closed system is no less than $\delta Q / T$, namely the Clausius inequality, $\mathrm{dS} \geq \delta \mathrm{Q} / \mathrm{T}$, where $\delta \mathrm{Q}$ denotes the amount of heat the outside gives to the target system, and $\mathrm{T}$ denotes absolute temperature.

Microscopically and statistically, entropy quantifies the number of microscopic configurations termed microstates, using the Boltzmann formula, $\mathrm{S}=\mathrm{K} \times \mathrm{Ln} \Omega$, where $\mathrm{S}$ denotes entropy, and $\mathrm{K}$ denotes Boltzmann constant, and $\Omega$ denotes the number of microstates. The entropies of perfect crystals of pure materials at absolute zero temperature are zero $(\Omega=1)$. This formula established by Ludwig Boltzmann suggests that when a system has fewer microstates, its entropy declines, and vice versa.

\subsubsection{The Formula of Gibbs free energy $[15,16]$}

According to the four laws of thermodynamics given above, particularly the second law of thermodynamics, the formula of Gibbs free energy was established, $\Delta \mathrm{G}=\Delta \mathrm{H}-\mathrm{T} \times \Delta \mathrm{S}$, where $\Delta \mathrm{G}$ denotes increase of Gibbs free energy, and $\Delta \mathrm{H}$ denotes increase of enthalpy, and $\mathrm{T}$ denotes absolute temperature, and $\Delta \mathrm{S}$ denotes increase of entropy.

Simply speaking, the formula of Gibbs free energy tells us that high-energy entities shall spontaneously release energy and thus become low-energy entities at a relatively low temperature (like the fact that water becomes ice at the temperature below zero degree centigrade), and low-energy entities shall spontaneously absorb energy and thus become high-energy entities at a relatively high temperature (like the fact that ice becomes liquid and gaseous water at 20 degrees centigrade).

\subsubsection{Features of CBEs [17,18]}

By mass, carbon is the fourth most abundant element in the universe after hydrogen, helium, and oxygen, and carbon is the 15th most abundant element in the Earth's surface. Carbon is unique in sharing electrons with multiple atoms to form covalent chemical bonds and soluble polymers at the temperatures commonly encountered on Earth. Most of the carbon-related covalent chemical bonds and other binding force inside CBEs are relatively weak, and thus can be destroyed by fires and other violent factors. 


\subsubsection{Formation and degradation of HHCBEs $[1,2]$}

At a relatively high temperature, as per the formula of Gibbs free energy and features of CBEs, LHCBEs spontaneously tend to absorb more energy to form HHCBEs. On the other side, these HHCBEs shall degrade sooner or later due to action of some inner or outer factors which can destroy carbon-related covalent chemical bonds or other binding forces inside HHCBEs. Moreover, as per the formula of Gibbs free energy, at a relatively low temperature, HHCBEs spontaneously tends to release energy and degrade to LHCBEs, like the fact that liquid water spontaneously tends to release energy and become ice at -20 degrees centigrade.

\subsubsection{Functions of CBEs changing spontaneously due to changes in structures and hierarchies of CBEs}

Functions of CBEs change spontaneously due to changes in structures and hierarchies of CBEs, and particularly, HHCBEs have spontaneously some functions unable to be fulfilled by LHCBEs [1,2]. This is also equal to the phrase that the whole is greater than the sum of its parts. For example, some proteins catalyze certain chemical reactions which cannot be fulfilled by any amino acids, and some animals have the function of language which cannot be fulfilled by any cells.

\subsubsection{Hierarchies of CBEs increase stepwise}

HHCBEs are formed hierarchy by hierarchy [1,2]. For example, amino acids, nucleotides and other middle organic molecules could not bypass the intermediate hierarchy of large organic molecules to form unicellular organisms, and large organic molecules could not bypass the intermediate hierarchy of unicellular hierarchy to form multicellular organisms.

\subsubsection{HHCBEs frequently change discontinuously}

In genetics or epigenetics, or in phenotype, HHCBEs frequently change discontinuously. An organism can change its genomic sequences, such as insertion with one or more genes, chromosomes, or a whole genome, through only one event, and thus greatly changes its phenotypic traits without any intermediate steps [2,3-5,19,20]. Moreover, a small genetic or epigenetic change, such as insertion, substitution, or methylation of a single nucleotide can greatly change a trait without any intermediate steps [3-5,21-23].

\subsection{Validation of the CBEET}

All the major views of the CBEET were validated through one or two ways at Sections 3-8: one is whether the views can explain some phenomena not well explained previously, and the other is whether the views reflect the reality better. Streamline application of these views in the whole evolutionary process described at Sections 5-8 also validated the CBEET.

\section{The driving force of evolution}

\subsection{Previous views (background)}

It has long been presumed that natural selection, mutation, genetic drift, or competition is the driving force of the natural process [3-5,24-26]. However, like the fact that the driving force for a car to run is from energy consumption, the driving force of evolution should rely heavily on energy. All the driving forces mentioned above are not directly related to energy, and in terminology, they are largely mechanisms of evolution which will be interpreted below.

\subsection{Driving force from thermodynamics (deduction)}

The Earth's surface has permanent, widespread, abundant, and temperate energy flow from sunlight and geotherm. The Earth is a rare habitable planet in astronomy [27]. It is in a suitable orbit and receives neither too strong nor too weak sunlight, which leads to temperate energy flow on Earth for billions of years. Many sites on Earth have emitted geothermal energy for millions or billions of years, particularly at hydrothermal vents [28,29]. The huge amount of water on Earth further regulates solar and geothermal energy through evaporation, diffusion, and rainfall, making it more temperate, lasting longer, and distributed more widely.

With permanent, widespread, abundant, and temperate energy flow on Earth, CBEs, as low-energy matter under relatively high temperature caused by the energy flow, tend to absorb more energy, to form HHCBEs, according to the thermodynamic formula of Gibbs free energy given at Section 2.2.3 [15,16]. This is the driving 
force of evolution which has driven the evolution of CBEs for billions of years. This is not a hypothesis, but a conclusion deduced from thermodynamics. The driving force is also providing energy for growth of all the current organisms on Earth, as simplified by the example given in Figure 2, which was clearly stated by Ludwig Boltzmann who established the statistical basis of entropy (Section 2.2.2) in 1886 (see the last paragraph of Section 4.7). It can be seen from the fact that there are more organisms in tropical rainforests than in frozen areas, because the driving force is stronger in tropical rainforests, with the $\mathrm{T}$ value in the formula of Gibbs free energy, $\Delta \mathrm{G}=\Delta \mathrm{H}-\mathrm{T} \times \Delta \mathrm{S}$, higher in tropical rainforests, making $\Delta \mathrm{G}$ further less than 0 (suggesting the change is more spontaneous).

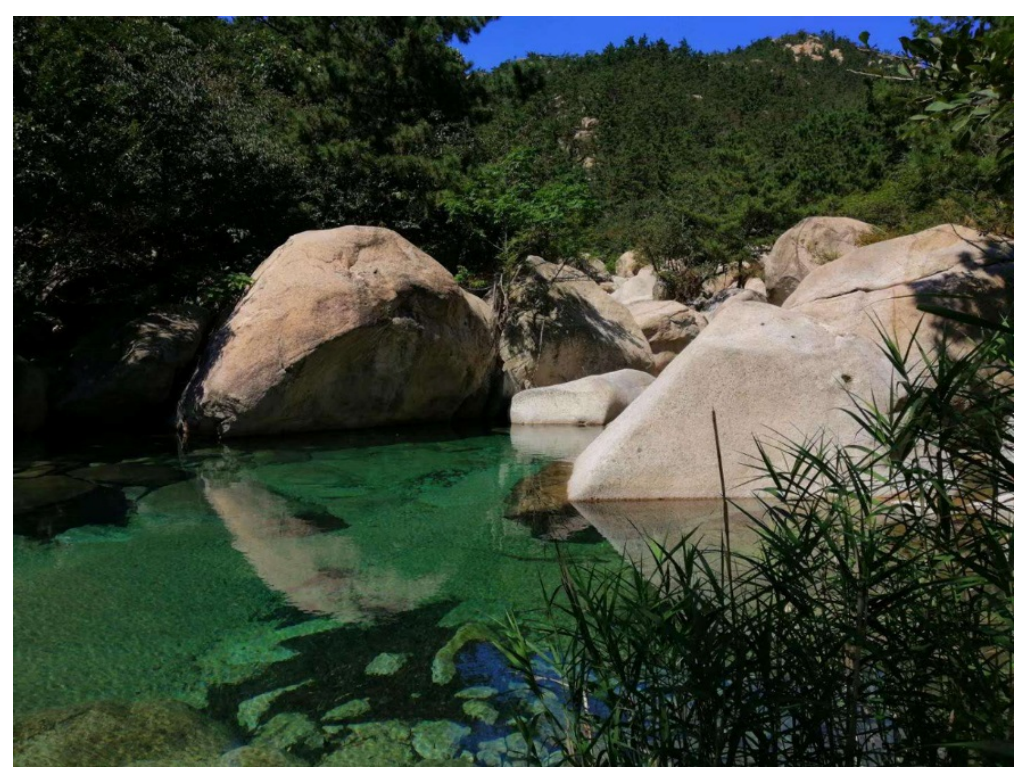

Figure 2. A picture of a pond under sunshine with spontaneous thermodynamic changes. As per the formula of

Gibbs free energy, under sunshine, stones shall spontaneously increase their temperature, and water shall spontaneously evaporate, and carbon-based entities (CBEs) in the plants shall spontaneously form into higherhierarchy CBEs, including synthesis of glucose, cellulose, proteins, and nucleic acids.

Initially, the driving force of evolution was from the energy of sunlight and geotherm, likely the energy at hydrothermal vents $[28,29]$. Later, with the increase of organisms on Earth, organisms not only harbor energy, but also contain materials suitable for reproduction of organisms, both of which can be employed to form other organisms. Therefore, biological energy became a source of the driving force of biological evolution (Figure 1). This is of paramount significance for animals which can actively obtain energy and materials from other organisms. As for humans, energy from coal, petrol, water flow, and even nuclear power has been utilized for the development of human society which in nature is an HHCBE system.

The relationship between CBE evolution and thermodynamics shall be further explained at Section 4.7. The driving force is important but insufficient for evolution. Whether some LHCBEs can absorb more energy to form HHCBEs is also determined by the inner organization of the LHCBEs, like the fact that energy consumption provides the driving force for a car, but whether the car can run is also determined by the inner organization of the car.

\subsection{Explanation of macroevolution (validation)}

This driving force of evolution can explain why non-living organic molecules evolved to lives, why unicellular organisms evolved to multicellular organisms, and why ectotherm animals evolved to warm-blooded animals. These three macroevolution events all helped CBEs on Earth to absorb more energy to form more HHCBEs. They cannot be explained with natural selection, because none of the processes added fitness to CBEs, as non-living CBEs could be fitter than unicellular organisms, and unicellular organisms could be fitter than 
multicellular organisms, and ectotherm animals could be fitter than warm-blooded animals [3-5,10,24,30].

During the billions of years of the Earth's history, in general, the amount and the diversity of HHCBEs including organisms on Earth are increasing [31]. However, meteorite impacts, huge volcano eruptions, long glacial periods, and other catastrophes can destroy the temperate energy flow on Earth or the inner structure of many organisms [32-35]. Consequently, the amount and the diversity of HHCBEs including organisms could decline greatly for the catastrophes, sometimes leading to mass extinctions [32-35].

\section{Progressive mechanisms of evolution}

\subsection{Previous views (background)}

Natural selection has been the central tenet of evolutionary biology since the nineteenth century. As per the early evolutionists represented by Charles Darwin, the fitness resulting from inheritable traits was accumulated through natural selection, a process usually expressed as the phrase of "survival of the fittest" in the fierce competition among too many progenies of an organism [1-4]. The phrase led to the advent of the theory of "social Darwinism" which was used to justify authoritarianism, eugenics, racism, imperialism, fascism, Nazism, and struggles between national or racial groups [36].

Later, in the middle of the twentieth century, Charles Darwin's evolutionary theory was reconciled with population genetics, leading to the Modern Synthesis of evolutionary theory [1-4]. The Modern Synthesis assumed that most mutations are harmful, and advantageous mutations usually have a small phenotypic effect, and could be incorporated into the population through the process of natural selection, which was not "survival of the fittest", but rather "gradual replacement with the fittest which carry advantageous mutations" [3-5]. Over long periods, many slight changes accumulate to yield large genetic and phenotypic differences among species.

In recent decades, the modern synthesis has been challenged by advances in multiple disciplines. Genomic researches suggested that natural selection is only one of the forces that shape genome evolution and is not quantitatively dominant [10,31]. Paleobiologic researches suggested that evolution of many species is in a punctuated equilibrium tempo with little change in long geological periods and significant change in short geological periods [1-7]. Epigenetic researches suggested that some acquired traits without genetic mutations are inheritable [3-5,11]. Microbiologic researches suggested that sometimes genetic mutations are not fully random [9]. Therefore, it is highly desirable to integrate with all these advances.

\subsection{Three progressive mechanisms (deduction)}

The progressive mechanisms of evolution, like the inner structure of a car, explain why the natural process has increased the complexity, hierarchy, functions, diversity, fitness of CBEs. As per the CBEET, there are three progressive mechanisms for evolution.

First, as shown in Figure 1, the organic synthesis driven by thermodynamics on Earth directly increases the amount of HHCBEs, and increases the structural complexity and hierarchy of CBEs, including from small molecules $\left(\mathrm{CO}_{2}, \mathrm{CH}_{4}, \mathrm{H}_{2} \mathrm{O}, \mathrm{H}_{2} \mathrm{~S}, \mathrm{NH}_{3}, \mathrm{Fe}, \mathrm{Zn}\right.$, etc.) to middle organic molecules (amino acids, nucleotides, glucose, acetyl coenzyme A, etc.), from middle organic molecules to large organic molecules (proteins, nucleic acids, polysaccharides, lipids, etc.), from large organic molecules to unicellular organisms, from unicellular organisms to multicellular organisms, and from multicellular organisms to groups including those of ants, bees, and humans.

Second, increased structural complexity and hierarchy of CBEs spontaneously offer complicated functions to CBEs, or to say, HHCBEs spontaneously have some functions unable to be fulfilled by LHCBEs (Section 2.2.6). For example, when some amino acids are transformed into a protein, the CBEs could obtain some novel functions which cannot be fulfilled by any amino acids, such as facilitating ions to pass lipid bilayer membrane (ion channels), emitting fluorescence, and facilitating transformation of adenosine diphosphate to adenosine triphosphate. Some unicellular organisms have the function of photosynthesis which cannot be fulfilled by any proteins. Some animals have the function of logic thinking which cannot be fulfilled by any cells. The structural complexity can adjust mutation rates in some organisms to adapt to environment changes, making mutations occur not fully randomly, as evidenced in evolution of microbial genomes and immunoglobulin genes [9,37]. 
Third, as elucidated below at Section 4.3, HHCBEs are regenerated with some changes under the action of the driving force of evolution. These regenerated HHCBEs with changes are under natural selection, leading to increase of diversity and increase of fitness of CBEs.

\subsection{The CBEET definition of natural selection (deduction)}

As per the CBEET, under the driving force from thermodynamics, LHCBEs tend to form HHCBEs and obtain novel functions. Sooner or later, the formed HHCBEs shall degrade into LHCBEs, due to the action of outer factors (e.g. fire burning) or inner factors (e.g. natural aging of CBEs) [1,2]. Therefore, lots of CBEs on Earth are in the cycle of formation and degradation of HHCBEs (See Section 2.2.5).

HHCBEs regenerated with the same CBEs through the same approach are usually somehow different due to the nature of organic synthesis, making changes in HHCBEs, including genomic mutations, epigenetic changes, and some uninheritable changes of organisms.

With these repeated cycles of degradation and regeneration of HHCBEs, some HHCBEs are formed faster and/or degrade more slowly, usually because of adequate driving force provided by the surroundings and adequate fitness due to good collaboration of their components. Some other HHCBEs are formed more slowly and/or degrade faster, usually because of inadequate driving force or inadequate fitness due to absence of good collaboration of their components. As per this difference, in the long run, some HHCBEs increase in quantity, and some other HHCBEs become extinct. This natural dynamic process is defined as natural selection in the CBEET (Figure 1).

Many HHCBEs existed on Earth in a small quantity for some time, particularly when they emerged not long time ago and when they encountered a catastrophe. Accordingly, if we say an HHCBE is fit in general or has adequate fitness, we mean that the HHCBE has not been extinct, which is equal to the claim that the HHCBE has passed natural selection in the past time. On the other side, if we say an HHCBE is unfit in general or has inadequate fitness, we mean that the HHCBE has been extinct, which is equal to the claim that the HHCBE has not passed natural selection in the past time. Therefore, natural selection in the CBEET is defined alternatively as survival of HHCBEs fit in general and elimination of HHCBEs unfit in general (Figure 1).

Whether an HHCBE is fit in general is determined not only by its inner structure (collaboration of its components), but also by its surroundings $[24,38]$. For example, great fitness in a hot rainforest can be a terrible burden in a cold desert.

\subsection{Comparison with previous definitions (validation)}

The CBEET definition maintains the core feature of the concept of natural selection in Charles Darwin's evolutionary theory and in the Modern Synthesis [3-5]: increase of fitness is because of natural survival of the fit and natural elimination of the unfit, making unfit HHCBEs survive less time and/or reproduce fewer progenies. On the other side, the CBEET definition is different from the previous definitions in multiple aspects.

First, the CBEET definition covers non-living CBEs and is thus more inclusive than previous definitions, and can be extended to chemical evolution for origin of life.

Second, the CBEET definition is equal to "survival of the ones fit in general". It thus allows co-existence of diversified variants with neutral changes. This definition is less harsh than "survival of the fittest" in Charles Darwin's theory, and more inclusive than "gradual replacement with the fittest which carry advantageous mutations" in the Modern Synthesis [1-4]. The CBEET definition is thus consistent with the notion of genetic drift and development of genomics, both of which suggested that most genomic changes are likely neutral without increase in fitness $[3-5,10,12,26]$. Moreover, the CBEET definition suggests that, when the environment is comfortable and far away from saturation with HHCBEs, the threshold of natural selection is low, which is beneficial for increase of the diversity of HHCBEs. On the other side, when the environment becomes dreadful, particularly in a catastrophe period, the threshold of natural selection is high, which can lead to mass extinction of organisms including those which were quite fit previously [32-35]. In a permanent and temperate environment without catastrophes, fitness of a species could become inadequate due to gradual changes of their biotic and abiotic surroundings, particularly when the environment is saturated with HHCBEs [24]. Additionally, we should differentiate replacement and diversification in evolution. Taxon replacement usually means that a taxon is unfit 
or has inadequate fitness, while taxon diversification means that two or more taxa from the same ancestor have adequate fitness.

Third, winners of natural selection as per the CBEET definition are determined by their overall fitness covering not only genetic mutations, but also inheritable epigenetic changes. This reflects the reality more comprehensively than previous definitions, because epigenetic changes are also important for organism to win natural selection.

Fourth, winners of natural selection as per the CBEET definition are determined by their overall fitness resulting from inheritable changes and uninheritable changes. This reflects the reality more comprehensively. For example, vaccination which is uninheritable makes many animals survive viral infections and pass the relevant natural selection. Uninheritable changes are covered in the CBEET definition, but they are excluded from previous definitions of natural selection.

Fifth, the CBEET definition of natural selection highlights "fit in general" or "overall fitness" because the reality is that the existence of a HHCBE is determined by its overall fitness, not by one of its advantages or disadvantages, although one of its traits may play a leading role in the overall fitness of an organism. Therefore, the CBEET allows an organism to have some disadvantageous traits, if its overall fitness related to reproduction and survival is enough. For example, antelopes are less strong than buffaloes to fight against carnivores, but they run fast and have other advantages, making them have adequate fitness in general.

Altogether, the CBEET definition of natural selection favors fitness and diversity, and is more inclusive and more comprehensive, and reflects the reality better in multiple aspects, than previous definitions. This, in turn, validates the CBEET definition.

\subsection{Positive selection and negative selection (extension)}

Organisms usually have accumulated much fitness through long geological periods. Therefore, most traits of organisms are under both positive selection (supporting those changes which can add fitness) and negative selection (inhibiting those changes which can reduce fitness) [39]. Natural selection is thus realized through the co-action of positive selection and negative selection.

Negative selection is evidenced by the fact that the first two nucleotides of most codons in the genomes of almost all organisms are conserved [25,39]. These two nucleotides have a much higher possibility than the third nucleotide of codons to make nonsynonymous changes. Moreover, because natural selection "selects" organisms as per their overall advantages consisting of all genomic sites and all traits, all genomic sites and all traits are under both positive selection and negative selection [39]. A conserved trait or a genomic site without change during a long geological period does not mean this trait or genomic site is not under natural selection, but rather under strong negative selection [39].

Moreover, a trait could add fitness in some aspects, but reduce fitness in other aspects for an organism, and a trait could reduce fitness in various aspects if it is out of a rational range. For example, proper increase of stature height could facilitate herbivores to identify and fight against carnivores, but makes herbivores unable to run fast, and too high herbivores are usually unfit in various aspects. This leads to the balance of the trait of stature resulting from the co-action of positive selection for the requirement of early identification of carnivores and negative selection for the requirement of running fast to avoid being captured. This example applies to many traits and sites in genomics [39]. Accordingly, natural selection works extensively in evolution.

\subsection{Natural selection and the driving force (discussion)}

As per the above deduction, the driving force of evolution deduced from thermodynamics provides energy for the evolution of CBEs. Moreover, the first two progressive mechanisms for evolution are directly derived from the driving force of evolution, and the third progressive mechanism (i.e. natural selection) is partially deduced from the driving force of evolution (Figure 1). Therefore, the driving force of evolution plays the first leading role in evolution, and natural selection is second to the driving force of evolution, although it remains a leading role in evolution. 
The role of energy in biological evolution has been highlighted [40,41], but energy has never been associated with the driving force of evolution, and the driving force of evolution from thermodynamics has never been assumed to be more important than natural selection.

\subsection{Order, evolution and thermodynamics (extension)}

The above three progressive mechanisms of evolution lead to increase of order in biology which includes the inner complicated structure and coordinated movement of many molecules. Order in biology is the basis of fitness of organism, and it is achieved through natural selection of millions or billions of years by eliminating those organisms with inadequate fitness.

The driving force of evolution is deduced from the formula of Gibbs free energy. The formula is derived from the second law of thermodynamics which indicates that entropy increases spontaneously in an isolated system, and sometimes entropy represents as chaos in physics. Thus, the fact that evolution leads to increase of order in biology seems largely contrary to the second law of thermodynamics leading to increase of entropy or chaos in physics $[15,16,42]$. Many scientists including Nobel laureates Erwin Schrödinger and Ilya Prigogine have tried to solve this conundrum which is also termed Schrödinger's paradox [42]. Most of them assumed that an organism is a system with low entropy, and it keeps low entropy through metabolism which absorbs low-entropy matter and discharges high-entropy matter, and thus the notion of "negative entropy" was established [42].

Soon after Erwin Schrödinger created the notion of "negative entropy", he met dramatic criticism from physicists [42]. He thus stated, "if I had been catering for them alone I should have let the discussion turn on free energy instead." This statement showed that he knew that "negative entropy" is not scientific, but he was reluctant to abandon it. Interestingly, he correctly pointed that [Gibb's] free energy, which is employed in this article, is suitable for this discussion.

The negative entropy notion is wrong in this field for the following reasons. As per the entropy formulas of Clausius (Section 2.2.2), order in biology showing a moving and warm feature with increased microstates, is largely contrary to order in physics which represents a static and cold with reduced microstates, as evidenced by the fact that crystals at zero degree of absolute temperature have the highest order and the lowest entropy in physics. For example, as per the Clausius inequality (Section 2.2.2), a lizard (or an ice cream) increases its entropy when it absorbs energy from sunlight. This is consistent with the Boltzmann formula because the number of microstates of the lizard (or an ice cream) increases when it absorbs energy from sunlight. Another example is that the order in biology of a dog declines, and the order in physics of the same dog increases, when the dog is dying in the snow with heat losing from the body.

Biological order is established through billions of years' evolution, rather than a short time input of "negative entropy". This is another error of the negative entropy notion. Contrary to the claim of Erwin Schrödinger, what we eat is not "negative entropy", but just food providing us with energy and nutrients, without much relationship to the order in biology encoded by our genomes. Interestingly, although Erwin Schrödinger created the notion of "negative entropy", he correctly postulated, before the discovery of the functions of nucleic acids, the existence of some molecules in cells encoding the order in biology [42]. This postulation, in turn, collided with the notion of "negative entropy".

Although evolution is complicated in many aspects, it is generally within the fields of Newtonian mechanics, organic chemistry, and thermodynamics. It thus complies with relatively simple physical and chemical laws. We need not employ some elusive concepts or novel laws for evolution [13,43]. However, it is tough for scientists to harmonize laws of thermodynamics and evolution as per the notion of "negative entropy" because the notion is wrong.

As per the CBEET, evolution is simple in physics or chemistry if we have recognized the fact that order in biology is largely contrary to order in physics, or the fact that entropy cannot be simply hooked with order [1,2]. High-speed traffic flow, large-scale choruses, and normally beating hearts all demonstrate some kinds of order with high entropy, contrary to the low-entropy status like a cold, static, and pure crystal.

A lecture of Ludwig Boltzmann, a founder of the concept entropy, addressed in 1886 to a formal meeting of the Imperial Academy of Science, had the following statement [44], "The general struggle for existence of animate beings is therefore not a struggle for raw materials - these, for organisms, are air, water and soil, all abundantly 
available - nor for energy which exists in plenty in any body in the form of heat (albeit unfortunately not transformable), but a struggle for entropy, which becomes available through the transition of energy from the hot sun to the cold earth. In order to exploit this transition as much as possible, plants spread their immense surface of leaves and force the sun's energy, before it falls to the earth's temperature, to perform in ways as yet unexplored certain chemical syntheses of which no one in our laboratories has so far the least idea. The products of this chemical kitchen constitute the object of struggle of the animal world." Although this statement was not directly linked to the driving force of evolution, but it provided the same thermodynamic view of this article. Unfortunately, this statement has not been paid enough attention by scientists. Moreover, the word "negative" was added intentionally and erroneously before the word "entropy" in this statement in Wikipedia [45], demonstrating that the notion of "negative entropy" has poisoned too many scientists. Brooks and Wiley in the 1980s accepted the notion of "negative entropy", and thus they had to employ a series of ambiguous concepts to deduce that entropy led to evolution [46].

\section{Application in chemical evolution}

Chemical evolution is also termed abiogenesis or origin of life, which is one of the most important and the most enigmatic topics in science. The concept and relevant theories regarding chemical evolution were first proposed by Alexander Oparin [47]. Many aspects of chemical evolution have been investigated in recent decades using experimental and theoretic tools from biology, chemistry, geology, and astronomy [3-5]. It has been revealed that meteorites could bring lots of organic molecules to the early Earth, including polycyclic aromatic hydrocarbon (PAH), amino acids, sugar, adenine, and guanine [48]. Also, billions of tons of these middle organic molecules could be formed from small organic or inorganic molecules, e.g. ammonia, methane, carbon dioxide, and hydrogen sulfide, particularly at volcanic hot springs and hydrothermal vents on the Earth [28,29,47]. Moreover, the possible routes for the formation of large organic molecules, e.g. proteins, DNA, RNA, polysaccharides and lipids at the early Earth have been widely explored [49]. However, the processes and mechanisms for many organic molecules to combine to form the first batch of lives remain enigmatic, although the RNA world, hypercycle, collectively autocatalytic sets, and other evolutionary hypotheses have been proposed [50,51].

\subsection{The process of the evolution of CBEs}

As elucidated above, the driving force of chemical evolution is from thermodynamics which leads to spontaneous synthesis of HHCBEs. As given in Section 2.2.7, HHCBEs are formed hierarchy by hierarchy. For example, amino acids, nucleotides and other middle organic molecules could not bypass the intermediate hierarchy of large organic molecules to form unicellular organisms, and large organic molecules could not bypass the intermediate hierarchy of unicellular hierarchy to form multicellular organisms. Accordingly, there should be the following seven major steps constitute the whole evolution of CBEs on Earth (Figure 1).

Step 1, some small molecules $\left(\mathrm{CO}_{2}, \mathrm{CH}_{4}, \mathrm{H}_{2} \mathrm{O}, \mathrm{H}_{2} \mathrm{~S}, \mathrm{NH}_{3}, \mathrm{Fe}\right.$, $\mathrm{Zn}$, etc.) absorbed energy and formed middle organic molecules (amino acids, nucleotides, glucose, acetyl coenzyme A, etc.). This step also occurred in other planets, and many tons of middle organic molecules were sent to Earth through meteorites [48]. The synthesized middle organic molecules were under natural selection because they were produced and maintained at different rates [52].

Step 2, some middle organic molecules absorbed energy and formed large organic molecules (proteins, nucleic acids, polysaccharides, lipids, etc.). The synthesized large organic molecules were under natural selection because they were produced and maintained at different rates. Since there were no templates or mechanisms to direct the synthesis of large organic molecules according to certain orders at this step, many kinds of proteins, nucleic acids, lipids, polysaccharides were thus produced, providing abundant candidates for forming HHCBEs in the coming steps.

Step 3, some large organic molecules collaborated with each other and formed large organic molecule aggregates (LOMAs), including the aggregates of lipid bilayer membrane and the aggregates allowing ions to pass lipid bilayer membrane [53]. From this step on, energy is not always required to form chemical bonds, but is required for the movement of CBEs to collaborate with each other. The formed LOMAs were under natural selection because they were produced and maintained at different rates. 
Step 4, some LOMAs collaborated with each other and formed complexes of LOMAs including the units for photosynthesis or the units for synthesis of proteins or nucleic acids. The formed complexes of LOMAs were under natural selection because they were produced and maintained at different rates.

Step 5, some complexes of LOMAs collaborated with each other and formed the first batch of unicellular organisms, which were the units having the complicated functions of self-replication via autocatalysis (for efficient generation of HHCBEs) and self-protection (for maintaining HHCBEs). The formed unicellular organisms were under natural selection because they were produced and maintained at different rates. Formation of unicellular organisms through the complicated functions of self-replication via autocatalysis and self-protection involved synthesis or formation of middle organic molecules, large organic molecules, LOMAs, complexes of LOMAs, and CBEs at these hierarchies were also under natural selection along with unicellular organisms. If a type of unicellular organisms is favored by natural selection, these CBEs involved in the formation of the unicellular organism are also favored by natural selection.

Step 6, some cells collaborated with each other and formed multicellular organisms, which include fungi, plants and animals. The formed multicellular organisms were under natural selection because they were produced and maintained at different rates. Meanwhile, CBEs below the hierarchy of multicellular organisms were also under natural selection along with multicellular organisms, if they were involved in the formation of multicellular organisms.

Step 7, some animals collaborated with each other and formed animal groups, which include societies of bees, ants, and humans. The formed animal groups were under natural selection because they were produced and maintained at different rates. Meanwhile, CBEs below the hierarchy of animal groups, including individual organisms and genes, were also under natural selection along with animal groups, if they were involved in the formation of animal groups.

Steps 1-5 constitute chemical evolution leading to origin of life; steps 5-6 constitute biological evolution in a narrow sense. Steps 6-7 constitutes group evolution including the development of human society (Figure 1).

The above steps were inevitable through backstepping of the process for the evolution of CBEs. For example, to form the first batch of unicellular organisms, many kinds of complexes of LOMAs should have been generated in large quantities on Earth, so that they could provide enough candidate combinations to obtain the complicated functions of self-replication via autocatalysis and self-protection at a very tiny possibility.

\subsection{The steps of chemical evolution}

The five steps constituting chemical evolution increased the structural complexity of HHCBEs. The increased structural complexity spontaneously offered novel complicated functions to HHCBEs, and finally led to origin of the first batch of lives, which had the complicated functions of self-replication via autocatalysis and self-protection. Compared to non-living HHCBEs, lives were much more efficient in absorbing energy and synthesis of HHCBEs.

The five steps constituting chemical evolution suggest that, before origin of the first batch of lives, there were five successive and overlapping worlds: the world of small molecules, the world of middle organic molecules, the world of large organic molecules, the world of LOMAs, the world of complexes of LOMAs, and the world of preliminary lives. Those preliminary lives could facilitate greatly the production, diversification, and optimization of HHCBEs, with their complicated functions of self-replication via autocatalysis and self-protection. This, in turn, could facilitate formation of novel preliminary lives with more fitness, and finally, this process led to the origin of the last universal common ancestor [54].

Compared with previous views [50,51], the CBEET highlights collaboration of various molecules/factors and stepwise increase of hierarchies of CBEs in chemical evolution. The CBEET underlines neither the effect of RNA nor some small or middle organic molecules with the function of autocatalysis.

\section{Application in biological evolution}

Biological evolution in its narrow sense was extended from and overlapped with chemical evolution. The driving force and the progressive mechanisms of biological evolution have been elucidated above. The following will discuss four topics of biological evolution: sexual reproduction, sexual selection, evolutionary tempo, and speciation mechanisms. 


\subsection{Sexual reproduction}

Sexual reproduction is a trait or complicated function, and is fulfilled when the complexity of an organism is complicated enough. Sexual reproduction has an important advantage over non-sexual reproduction, as it can increase genetic diversity safely through recombination of many genes that have passed natural selection for many generations [55]. Increased genetic diversity is useful for organisms to adapt to various environments or environmental changes. This can avoid generating changes using genomic mutations which are usually harmful.

\subsection{Sexual selection}

When the structures of some organisms have been complicated enough, the function of sexual selection is fulfilled, which adds another advantage to sexual reproduction: it allows those stronger or healthier ones to reproduce more progenies, which is helpful to keep or increase fitness of the species and helpful for the species to maintain adequate fitness and pass natural selection. Sexual selection is a trait under natural selection, and this trait can greatly shape evolution of many taxa due to its active selection [3-5].

\subsection{Evolutionary tempo}

Evolution of many species has shown in a punctuated equilibrium tempo with little change in long geological periods interrupted by short geological periods with significant changes [3-5]. There could be two reasons for this evolutionary tempo. First, as given in Section 2.2.8, organisms could change greatly in phenotype due to only one genetic or epigenetic change, and an organism carrying a significant change in phenotype can pass natural selection if it has adequate fitness. Second, if a trait in phenotype is inheritable, and the trait changes gradually generation by generation, and the change is favored by positive selection or sexual selection, then a significant change can be accumulated within a short geological period (e.g. increase by $1 \mathrm{~cm}$ in stature per generation can lead to increase by $1 \mathrm{~m}$ in stature in 100 generations which can be fulfilled in a short geological period). When the change in this trait shall not add fitness in general to the taxon, the trait shall maintain stable for a long geological period due to negative selection (see the last paragraph of Section 4.5), demonstrating the tempo of punctuated equilibrium (Figure 3). Previously, the tempo of punctuated equilibrium was interpreted using geographic isolation [3-5], and here the CBEET provides a novel rational explanation for the tempo of punctuated equilibrium based on natural selection, which covers not only the effect of geographic isolation but also the effect of temporal changes (e.g. climate changes).

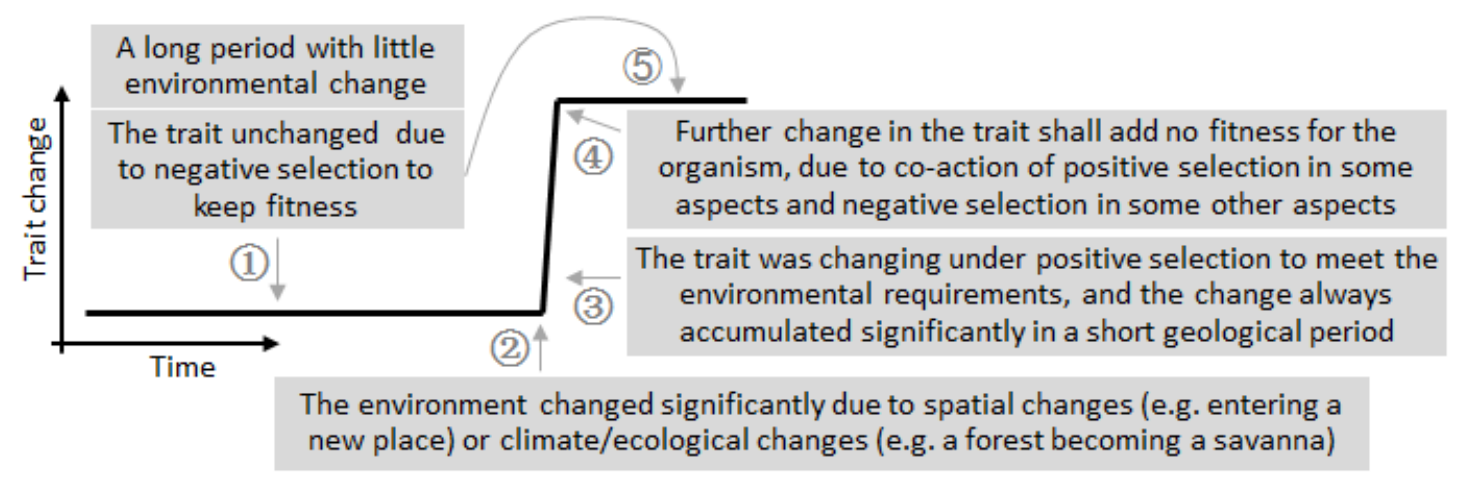

Figure 3. Reasons of the punctuated equilibrium tempo exhibited in the evolution of many organisms

\subsection{Sympatric speciation}

Allopatric speciation is important and easily comprehensible, while sympatric speciation is less comprehensible, and the frequency with which sympatric speciation occurs is not known [56]. Different traits are required to obtain different sources of energy and food in the same area. This can result in sympatric speciation, and likely led to sympatric speciation in some insects that become dependent on different host plants in the same area. 
The CBEET underlines overall fitness rather than a single trait. This suggests a novel mechanism of sympatric speciation, because multiple combinations of traits can all have adequate fitness in obtaining the same source of energy and food, or occupying the same ecological niche in the same area. This could lead to sympatric speciation of buffalos and antelopes, both of which rely on grazing and avoiding being killed by carnivores. Buffalos employ the combination of large stature and great defensive capability, while antelopes employ the combination of small stature and rapid running capability.

Sexual selection could facilitate the process of sympatric speciation through the above two mechanisms, because sexual selection can fix and enlarge the relevant changes [55].

When a big niche has abundant temperate energy and materials for increase of HHCBEs, and the HHCBEs in the niche has been far away from saturation, particularly when a HHCBE has made a breakthrough in its structure or obtained a greatly useful trait or function, it can spark an explosive adaptive radiation in the big niche (e.g. Cambrian explosion) $[14,20]$. Under such scenarios, it is easy for an HHCBE to have adequate fitness, through various combinations of multiple traits, and sympatric speciation is thus facilitated.

\section{Application in group evolution}

Group evolution was extended from and overlapped with biological evolution. The major role in biological evolution for direct collaboration and struggles for energy and materials is individuals of organisms, while the major role in group evolution for direct collaboration and struggles for energy and materials involves groups of animals.

An individual cell can be considered as a group of molecules, many of which collaborate closely and show altruism to others (altruism is defined as the action supporting the existence and reproduction of other entities). An animal can be considered as a group of cells many of which collaborate closely and show altruism to others. This extrapolation helps us to understand the emergence, existence, and evolution of animal groups which harbor a few to millions of individuals, many of which collaborate closely and show altruism to others. Through this extrapolation, altruism is comprehensible because it provides the basis of fitness for HHCBEs throughout the CBE evolution. No altruism means no collaboration and no fitness. Previous evolutionary theories have not revealed the driving force of evolution, and sometimes they explain animal behaviors including altruism using the tenet of selfishness of genes or inclusive fitness [57]. The tenet of selfishness of genes or inclusive fitness is largely rational also because they are consistent with the spontaneous increase of HHCBEs driven by thermodynamics.

Many species of animals have formed groups, and these animals include ants, bees, spiders, buffaloes, elephants, dogs, lions, and humans. Individuals in groups of bees, lions, and humans are willing to sacrifice themselves for fighting against invaders. Many mammals care for their progenies for months or years. These are typical types of collaboration in animal groups.

As a hierarchy higher than individual organisms, animal groups have novel functions which cannot be fulfilled by individual organisms. For example, some ant groups can have agriculture (planting fungi for food) or husbandry (raising aphids for collecting honeydew as food) [58]. This is consistent with the driving force of animal evolution which is the tendency to obtain more energy from other species of organisms. Another important advantage of animal groups in fitness is that struggles among individuals of the same species living closely are somehow avoided.

Different species of animals have developed different levels of groups. The highest level of groups is termed eusociality, and the relevant animals are termed eusocial animals, of which some individuals reduce their own lifetime reproductive potential to raise the offspring of others [59]. If an animal taxon shows a degree of sociality beyond courtship and mating, but lacks any of the characteristics of eusociality, it is said to be presocial. Presocial groups include the families of sparrows, antelopes, and lions. Although presocial species are much more common than eusocial species, eusocial species have disproportionately large populations, which supports the above notion that altruism and collaboration inside groups can add fitness to eusocial animals.

Besides altruism and collaboration, struggles are also crucial to maintain animal groups. Inner struggles in animal groups include the combat for obtaining mating opportunities and killing some members for food when food is in critical shortage. Outer struggles are also widespread because animals rely on energy and materials from other organisms, like the fact that lions kill and eat antelopes for food. Inner collaboration of animal groups 
increases greatly the ability to win the outer struggles. Sometimes, inner collaboration can avoid inner struggles within animal groups, and this could add great fitness to the group. For example, it has been observed that several male lions collaborated closely to defeat an old lion king, and they shared the mating opportunities, and collectively fought against invaders. These several male lions could have no mating opportunities without the inner collaboration.

Scenarios of oppression (a kind of struggles) widely exist in the same group of lions, monkeys, mice, and humans, particularly when there is a significant difference in power among individuals. Meanwhile, scenarios of anti-oppression also widely exist in these groups. Anti-oppression events usually lead to expelling or killing of the failed side. The peace and collaboration within animal groups are thus interrupted with these anti-oppression events. Sometimes oppression is too risky and costly, particularly when the oppressed individuals become powerful. This explains the decline of oppression in human society in recent centuries with the ending of most feudal societies and colonial rulings, because the oppressed people have become more powerful. Decline in oppression is important for humans to collaborate to obtain more energy and materials for the development of human society.

In functions, animals rely on free movement for their existence and making contribution to their groups. In evolution, animals rely on changes for making diversity and adapting to the environment. These two aspects highlight the importance of freedom. On the other side, almost all members within animal groups should obey some rules, which is fundamental for collaboration within animal groups. Lion kings should obey some rules to take their responsibility to fight against invaders. Worker bees should obey some rules to work diligently all days for their groups. Drivers should obey traffic rules. Similarly, molecules should obey some rules with some freedom in cells, and cells should obey some rules with some freedom in multicellular organisms. Therefore, obeying rules with balanced freedom is embodied throughout the evolution of CBEs.

\section{Suggestions for human society}

Evolutionary views have influenced human activities and development of human society for decades [15,33,59,60]. However, previous evolutionary theories overestimated the phenomenon of selfishness, the importance of competition, and elimination of those less fit in certain traits [1-5,33]. They underestimated the increase of hierarchy, increase of diversity, importance of altruism and collaboration, and resistance power of the oppressed individuals or groups. They thus exerted negative influence on the development of human society. Socialists had to establish separate theories, such as the cultural evolution theory, to advocate altruism and collaboration for harmonious development of human society [61,62].

In contrast, the CBEET, which is more comprehensive than previous evolutionary theories, provides comprehensive and constructive suggestions for the development of human society. First, as per the CBEET, natural selection allows co-existence of many differences and changes if they are of adequate fitness. This suggests that we should respect diversity in races, culture, and management systems. Second, natural selection accepts the effects of endeavor even if the effects are uninheritable. This suggests that we should work hard to overcome some difficulties and increase our fitness. Third, natural selection requires overall development and overall advantages, rather than a single trait. This is important for human individuals and human society in making decisions. Fourth, human society is in nature animal groups, and animal group evolution favors altruism and collaboration as well as underlines struggles, and some animals have employed inner collaboration to reduce inner struggles. This is of paramount importance for human society, as altruism and collaboration are critical for winning and avoiding struggles. Fifth, the CBEET suggests that oppression in human society should be reduced as the oppressed humans have become more powerful than the past. Sixth, obeying rules with balanced freedom is fundamental throughout the evolution of CBEs. The last three views collectively suggest that humans should strengthen altruism and collaboration to struggle for peace, balanced freedom, and rational social norms. They can thus vindicate human rights which have not been convincingly proved with previous biological evolutionary theories. On the other side, these views of human rights and social norms have been markedly strengthened through education and encouragement of humans, demonstrating the effect of the cultural evolution which is unique in humans and expresses natural principles of the evolution of CBEs [62].

Besides meteorite impacts, super volcano eruptions, major climate changes, infectious diseases, unscientific beliefs, and rapid increase of global population jeopardizing human society [63], a leading challenge to the current 
human society is that automatic machines are taking away too many job opportunities, which aggravates wealth inequality. Again, as per the CBEET, collaboration, rather than the outdated strategies of invasion, looting, or oppression, is critical to solving these conundrums, which requires profound rethought and reform of various social notions and systems, particularly those greatly reducing national or international collaboration.

The above suggestions of the CBEET are different from social Darwinism which was established on a prejudiced evolutionary model. They are important for harmonious development of human society. Therefore, the CBEET bridges natural sciences and social sciences and sheds novel insights into harmonious development of human society.

\section{Conclusions}

A novel evolutionary theory termed the CBEET is extrapolated from observations and deduced from thermodynamics in this article. In one sentence, the CBEET can be expressed as "Evolution which favors fitness and diversity is driven hierarchy-wise by energy".

The CBEET reveals the driving force of evolution using thermodynamics and establishes the driving force as the first leading role in evolution, which can explain why and how the hierarchy, complexity, functions, fitness, and order of CBEs increase on Earth. The CBEET is thus a live theory because it is based on energy and powerful in explaining various evolutionary conundrums.

The CBEET redefines natural selection, and reestablishes its key role in evolution. The redefined natural selection is more inclusive and works more extensively. It reflects realities better than previous theories and harbors advances in molecular evolution, genomics, and microbiology achieved in recent decades.

The driving force and natural selection described by the CBEET apply not only to biological evolution, but also to chemical evolution and group evolution including the development of human society.

The CBEET removes several elusive or erroneous evolutionary views including those pertaining to negative entropy, survival or replacement of the fittest, and limited action of natural selection in molecular or genomic evolution. Meanwhile, it respects diversity, genetic drift, non-adaptive changes, and non-random mutations.

The CBEET integrates with research advances in biology, geology, chemistry, astronomy, and social sciences. The CBEET suggests that human society should respect diversity, underline endeavor and overall development, strengthen collaboration to struggle against multiple challenges jeopardizing human society, and strengthen collaboration to struggle for peace, balanced freedom, and rational social norms.

In conclusion, the CBEET is a live and powerful evolutionary theory. It reveals the driving force of evolution from thermodynamics and reestablishes the leading role of natural selection. It integrates with advances of multiple disciplines and provides plausible answers to multiple evolutionary conundrums. It removes several elusive or erroneous views and elucidates evolution in a more comprehensive and comprehensible way. It also sheds novel insights into harmonious development of human society.

Funding: This research has not been supported by any funds.

Acknowledgments: The author is grateful to Meng Yang and Jiwang Chen for providing valuable comments.

Conflicts of Interest: The author declares no conflict of interest.

\section{References}

1. Chen JM. A new evolutionary theory deduced mathematically from entropy amplification. Chin Sci Bul. 2000;45(1):91-96. https://doi.org/10.1007/BF02884912

2. Chen JM, Chen JW. Root of science: the driving force and mechanisms of the extensive evolution. Beijing, China: Science Press, 2000.

3. Futuyma DJ, Kirkpatrick M. Evolution. 4th ed. Sunderland, UK: Sinauer Press, 2017.

4. Huneman P, Walsh DM. Challenging the Modern Synthesis: adaptation, development, and inheritance. Oxford, UK: Oxford University Press, 2017. 
5. Preiner $\mathrm{M}$, Asche $\mathrm{S}$, Becker $\mathrm{S}$, et al. The future of origin of life research: bridging decades-old divisions. Life (Basel). 2020;10(3):20. http://doi.org/10.3390/life10030020

6. Pagel M. Natural selection 150 years on. Nature. 2009;457:808-811. https://doi.org/10.1038/nature07889

7. d'Ischia M, Manini P, Moracci M, et al. Astrochemistry and astrobiology: materials science in wonderland? Int J Mol Sci. 2019;20(17):4079. http://doi.org/10.3390/ijms20174079

8. Orgel L. In the beginning. Nature. 2006;439(7079):915-915. https://doi.org/10.1038/439915a

9. Fitzgerald DM, Rosenberg SM. What is mutation? A chapter in the series: How microbes "jeopardize" the modern synthesis. PLoS Genet. 2019;15(4): e1007995. https://doi.org/10.1371/journal.pgen.1007995

10. Koonin EV. Darwinian evolution in the light of genomics. Nucleic Acids Res. 2009;37(4):1011-1034. http://doi.org/10.1093/nar/gkp089

11. Dickins TE, Rahman Q. The extended evolutionary synthesis and the role of soft inheritance in evolution. Proc Biol Sci. 2012;279(1740): 2913-2921. http://doi.org/10.1098/rspb.2012.0273

12. Casillas S, Barbadilla A. Molecular population genetics. Genetics. 2017;205(3):1003-1035. https://doi.org/10.1534/genetics.116.196493

13. Walker SI. Origins of life: a problem for physics, a key issues review. Rep Prog Phys. 2017;80(9):092601. https://doi.org/10.1088/1361-6633/aa7804

14. Cooney C, Bright J, Capp E, et al. Mega-evolutionary dynamics of the adaptive radiation of birds. Nature. 2017;542:344-347. https://doi.org/10.1038/nature21074

15. DeVoe H. Thermodynamics and chemistry. 2nd Edition. Version 10. http://www2.chem.umd.edu/thermobook/v10-screen.pdf. Accessed on Sep. 20, 2020.

16. Borgnakke C, Sonntag RE. Fundamentals of Thermodynamics. 8th edition. Hoboken, USA: John Wiley \& Sons, 2013.

17. Carbon. https://en.wikipedia.org/wiki/Carbon. Accessed on Sep. 20, 2020.

18. Marigo P, Cummings JD, Curtis JL, et al. Carbon star formation as seen through the non-monotonic initialfinal mass relation. Nat Astron. 2020;4:1-9. https://doi.org/10.1038/s41550-020-1132-1

19. Ermakova GV, Kucheryavyy AV, Zaraisky AG, et al. Discovery of four Noggin genes in lampreys suggests two rounds of ancient genome duplication. Commun Biol. 2020;3:501. https://doi.org/10.1038/s42003-02001234-3

20. McGee MD, Borstein SR, Meier JI, et al. The ecological and genomic basis of explosive adaptive radiation. Nature (2020). https://doi.org/10.1038/s41586-020-2652-7. Epub ahead of print.

21. Torres-Garcia S, Yaseen I, Shukla M, et al. Epigenetic gene silencing by heterochromatin primes fungal resistance. Nature. 2020;585:453-458. https://doi.org/10.1038/s41586-020-2706-x

22. Pillai AS, Chandler SA, Liu Y, et al. Origin of complexity in haemoglobin evolution. Nature. 2020;581:480-485. https://doi.org/10.1038/s41586-020-2292-y

23. Lin Z, Chen L, Chen X, et al. Biological adaptations in the Arctic cervid, the reindeer (Rangifer tarandus). Science. 2019;364(6446):eaav6312. http://doi.org/10.1126/science.aav6312

24. Benton MJ. The Red Queen and the Court Jester: species diversity and the role of biotic and abiotic factors through time. Science. 2009;323(5915):728-732. http://doi.org/10.1126/science.1157719

25. Hershberg R, Petrov DA. Selection on codon bias. Annu Rev Genet. 2008;42(1):287-299. http://doi.org/10.1146/annurev.genet.42.110807.091442

26. Nei M. Mutation-driven evolution. Oxford, UK: Oxford University Press, 2013.

27. Seager S. Exoplanet habitability. Science. 2013;340(577):577-581. http://doi.org/10.1126/science.1232226

28. Dodd MS, Papineau D, Grenne T, et al. Evidence for early life in Earth's oldest hydrothermal vent precipitates. Nature. 2017;543(7643): 60-64. http://doi.org/10.1038/nature21377. PMID 28252057.

29. Proskurowski G, Lilley MD, Seewald JS, et al. Abiogenic hydrocarbon production at lost city hydrothermal field. Science. 2008;319(5863):604-607. http://doi.org/10.1126/science.1151194. 
30. Lynch M. The frailty of adaptive hypotheses for the origins of organismal complexity. Proc Natl Acad Sci U S A. 2007;104(Suppl 1):8597-8604. http://doi.org/10.1073/pnas.0702207104

31. Rohde RA, Muller RA. Cycles in fossil diversity. Nature. 2005;434(7030):208-210. http://doi.org/10.1038/nature03339

32. Nozaki T, Ohta J, Noguchi T, et al. A Miocene impact ejecta layer in the pelagic Pacific Ocean. Sci Rep. 201;9(1):16111. http://doi.org/10.1038/s41598-019-52709-1.

33. Percival LME, Ruhl M, Hesselbo SP, et al. Mercury evidence for pulsed volcanism during the end-Triassic mass extinction. Proc Natl Acad Sci U S A. 2017;114(30):7929-7934. http://doi.org/10.1073/pnas.1705378114

34. Fields BD, Melott AL, Ellis J, et al. Supernova triggers for end-Devonian extinctions. Proc Natl Acad Sci U S A. 2020;117(35):21008-21010. http://doi.org/10.1073/pnas.2013774117

35. Bader J, Jungclaus J, Krivova N, et al. Global temperature modes shed light on the Holocene temperature conundrum. Nat Commun. 2020;11:4726. https://doi.org/10.1038/s41467-020-18478-6

36. Rudman LA, Saud LH. Justifying Social Inequalities: The role of social Darwinism. Pers Soc Psychol B. 2020;46(7):1139-1155. https://doi.org/10.1177/0146167219896924

37. Olivieri DN, Mirete-Bachiller S, Gambón-Deza F. Insights into the evolution of IG genes in Amphibians and Reptiles. Dev Comp Immunol. 2020: 103868. https://doi.org/10.1016/j.dci.2020.103868. Epub ahead of print.

38. McGee MD, Borstein SR, Meier JI, et al. The ecological and genomic basis of explosive adaptive radiation. Nature. 2020. https://doi.org/10.1038/s41586-020-2652-7

39. Chen J, Sun Y. Variation in the analysis of positively selected sites using nonsynonymous/synonymous rate ratios: an example using influenza virus. PLoS One. 2011;6(5):e19996. http://doi.org/10.1371/journal.pone.0019996.

40. Martin WF, Sousa FL, Lane N. Energy at life's origin. Science. 2014;344:1092-1093. https://doi.org/10.1126/science.1251653

41. Sousa FL, Thiergart T, Landan G, et al. Early bioenergetic evolution. Philos Trans R Soc Lond B Biol Sci. 2013;368(1622):20130088. http://doi.org/10.1098/rstb.2013.0088

42. Schrödinger E. What is life - the physical aspect of the living cell. Cambridge, UK: Cambridge University Press, 1944.

43. Nicholson SB, García-Pintos LP, del Campo A, et al. Time-information uncertainty relations in thermodynamics. Nat Phys. 2020. https://doi.org/10.1038/s41567-020-0981-y. Epub ahead of print.

44. Boltzmann L. The second law of thermodynamics. In Theoretical physics and philosophical problems (pp. 13-32). Dordrecht, Netherlands: Springer, 1974.

45. Entropy and life. https://en.wikipedia.org/wiki/Entropy_and_life. Accessed on Sep. 20, 2020.

46. Brooks DR, Wiley EO. Evolution as entropy. Chicago, USA: University of Chicago Press, 1988.

47. Lazcano A. Alexandr I. Oparin and the origin of life: A historical reassessment of the heterotrophic theory. J Mol Evol. 2016;83(5-6):214-222. http://doi.org/10.1007/s00239-016-9773-5.

48. Follmann H, Brownson C. Darwin's warm little pond revisited: from molecules to the origin of life. Naturwissenschaften. 2009;96(11):1265-1292. http://doi.org/10.1007/s00114-009-0602-1.

49. Patel BH, Percivalle C, Ritson DJ, et al. Common origins of RNA, protein and lipid precursors in a cyanosulfidic protometabolism. Nat Chem. 2015;7(4): 301-307. http://doi.org/10.1038/nchem.2202

50. Kauffman SA. Approaches to the origin of life on Earth. Life (Basel). 2011;1(1):34-48. http://doi.org/10.3390/life1010034

51. Robertson MP, Joyce GF. The origins of the RNA world. Cold Spring Harb Perspect Biol. 2012;4(5): a003608. http://doi.org/10.1101/cshperspect.a003608

52. Subramanian H, Brown J, Gatenby R. Prebiotic competition and evolution in self-replicating polynucleotides can explain the properties of DNA/RNA in modern living systems. BMC Evol Biol. 2020;20(1):75. http://doi.org/10.1186/s12862-020-01641-4 
53. Levy E, Erba E, Robinson C, et al. Assembly reflects evolution of protein complexes. Nature. 2008;453:1262-1265. https://doi.org/10.1038/nature06942

54. Weiss MC, Sousa FL, Mrnjavac N, et al. The physiology and habitat of the last universal common ancestor. Nat Microbiol. 2016;(9):1-8. https://doi.org/10.1038/nmicrobiol.2016.116

55. Hoekstra R. Why sex is good. Nature. 2005;434:571-573. https://doi.org/10.1038/434571a

56. Bird CE, Fernandez-Silva I, Skillings DJ, et al. Sympatric Speciation in the Post "Modern Synthesis” Era of Evolutionary Biology. Evol Biol. 2012;39:158-180. https://doi.org/10.1007/s11692-012-9183-6

57. Fehr E, Fischbacher U. The nature of human altruism. Nature. 2003;425:785-791. https://doi.org/10.1038/nature02043

58. Styrsky JD, Eubanks MD. Ecological consequences of interactions between ants and honeydew-producing insects. Proc Biol Sci. 2007;274(1607):151-164. http://doi.org/10.1098/rspb.2006.3701

59. Nowak M, Tarnita C, Wilson E. The evolution of eusociality. Nature. 2010;466:1057-1062. https://doi.org/10.1038/nature09205

60. Ferriere, R, Michod, R. Inclusive fitness in evolution. Nature. 2011;471:E6-E8. https://doi.org/10.1038/nature09834

61. Carroll SP, Jørgensen PS, Kinnison MT, et al. Applying evolutionary biology to address global challenges. Science. 2014;346(6207):1245993. https://doi.org/10.1126/science.1245993.

62. Henrich J. The secret of our success: how culture is driving human evolution, domesticating our species, and making us smarter. Princeton, UK: Princeton University Press, 2015.

63. Lufkin B. 50 Grand Challenges for the 21st Century. BBC. 2017. https://www.bbc.com/future/article/20170331-50-grand-challenges-for-the-21st-century. Accessed on Sep. $20,2020$. 